\title{
Recurrent Cholangitis with Congenital Hepatic Fibrosis and Pancreaticobiliary Maljunction after Roux-en-Y Reconstruction
}

\author{
Tokio Sugiura ${ }^{1}$ Takeshi Endo ${ }^{1} \quad$ Koichi Ito $^{1}$ Kenji Goto ${ }^{1}$ Yoko Sato ${ }^{2}$ Satoshi Kondo ${ }^{3}$ Tatsuya Suzuki ${ }^{4}$ \\ Takashi Hashimoto ${ }^{4}$
}

${ }^{1}$ Department of Pediatrics and Neonatology, Nagoya City University, Nagoya, Japan

2 Department of Pediatric Surgery, Nagoya City West Medical Center, Nagoya, Japan

${ }^{3}$ Department of Pediatric Surgery, Nagoya City University, Nagoya, Japan

${ }^{4}$ Department of Pediatric Surgery, Fujita Health University School of Medicine, Toyoake, Japan

Address for correspondence Tokio Sugiura, MD, PhD, Department of Pediatrics and Neonatology, Nagoya City University, 1 Kawasumi, Mizuho-cho, Mizuho-ku, Nagoya, Aichi 467-8601, Japan (e-mail: tokio@med.nagoya-cu.ac.jp).

Eur J Pediatr Surg Rep 2013;1:43-45.
Abstract
Keywords
- congenital hepatic fibrosis
- pancreaticobiliary maljunction
- cholangitis
- Roux-en-Y reconstruction
- liver transplantation

A 1-year-old girl had pancreaticobiliary maljunction, a choledochal cyst, and polycystic kidney. At the age of 4 years, she was treated by resection of the choledochal cyst and Roux-en-Y reconstruction because of the cyst's risk of cancer. She was diagnosed as having congenital hepatic fibrosis based on the histological findings. Postoperatively, she suffered recurrent fever of unknown origin, refractory to several antibiotics. At the age of 6 years, she underwent living donor liver transplantation from her father. Multidrug-resistant Pseudomonas aeruginosa was cultured in the recipient's liver. After liver transplantation, she had no episodes of recurrent fever. Roux-en-Y reconstruction should be avoided for ductal plate malformations such as congenital hepatic fibrosis.

\section{Introduction}

Congenital hepatic fibrosis (CHF) is a rare disorder of the portobiliary system characterized histologically by defective remodeling of the ductal plate, abnormal branching of the intrahepatic portal veins, and progressive fibrosis of the portal tracts. ${ }^{1}$ Typically, CHF is associated with autosomal recessive polycystic kidney disease. ${ }^{2}$ Although liver transplantation is curative, it should be limited to the minority of patients with chronic cholangitis or progressive hepatic dysfunction. In most instances, CHF is a disorder characterized by well-preserved hepatic function and a good prognosis if complications such as variceal bleeding and cholangitis are controlled. ${ }^{3}$

Pancreaticobiliary maljunction (PBM) is a congenital anomaly defined as a union of the pancreatic and biliary ducts outside the duodenal wall. PBM is associated with a high risk

received

December 30, 2012

accepted after revision

January 16, 2013

published online

March 19, 2013 for biliary tract carcinoma because of the continuous reflux of pancreatic juice into the biliary tract. ${ }^{4}$ To prevent biliary tract carcinogenesis in PBM patients, the cystic-dilated choledochus should be excised in childhood before the development to a precancerous stage. ${ }^{5} \mathrm{~A}$ case of CHF and PBM who suffered recurrent fever of unknown origin not responsive to several antibiotics after Roux-en-Y reconstruction is reported.

\section{Case Report}

A 1-year-old girl was referred to our hospital due to prolonged diarrhea. She had mild hepatosplenomegaly. Her kidneys were not palpable. Her height and weight were within the reference ranges, and her developmental history was ageappropriate. Her abdominal enhanced computed tomography

(c) 2013 Georg Thieme Verlag KG Stuttgart · New York
DOI http://dx.doi.org/ 10.1055/s-0033-1341420. ISSN 2194-7619. 


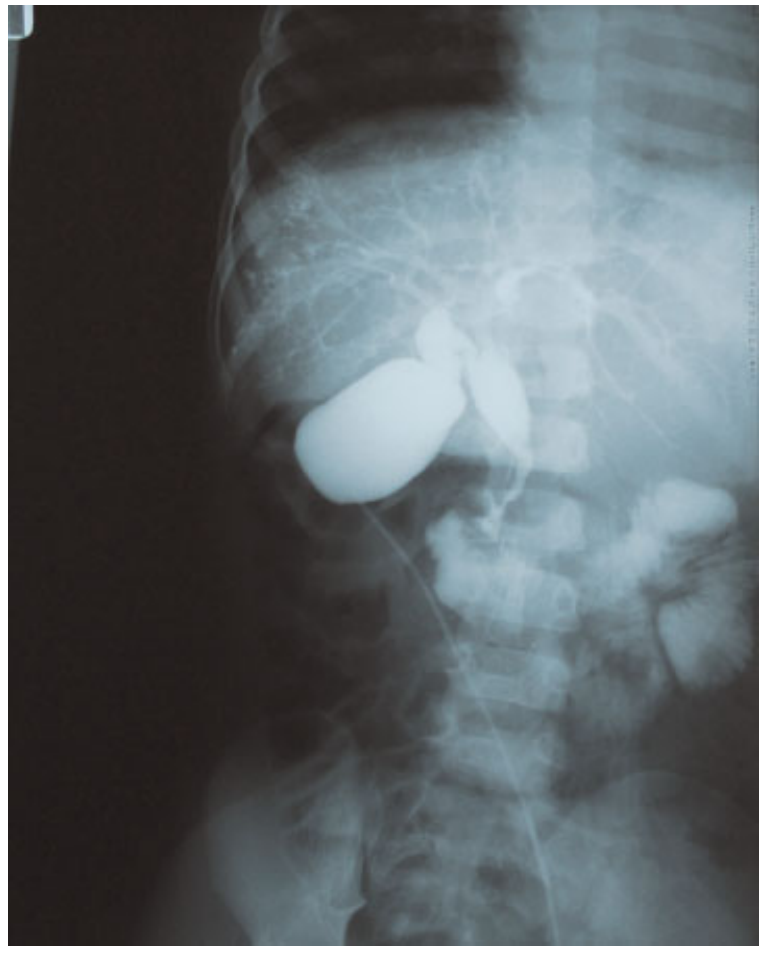

Fig. 1 Operative cholangiography shows pancreaticobiliary maljunction and a choledochal cyst.

(CT) showed hepatomegaly, dilation of intrahepatic bile ducts, and a polycystic kidney. Magnetic resonance cholangiopancreatography and drip infusion cholangiographic CT revealed PBM and a choledochal cyst. She had no variceal bleeding or jaundice. Viral markers of hepatitis A virus, hepatitis B virus, hepatitis C virus, cytomegalovirus, and Epstein-Barr virus were all negative. Her renal function was normal and renal biopsy was not performed. The parents were healthy, non-consanguineous Japanese. The patient had a healthy elder sister and younger brother, and her family had no liver or renal diseases.

At the age of 4 years, she was treated by resection of the choledochal cyst and Roux-en-Y reconstruction because of the cyst's risk of cancer. Operative cholangiography showed PBM and a choledochal cyst ( - Fig. 1). On liver biopsy specimen examination, portal areas greatly expanded by fibrous tissue and increased bile ducts were seen. The bands sometimes connected with adjacent portal tracts. Distorted irregular bile ducts in the portal tracts were noted ( - Fig. 2a). She was diagnosed as having CHF based on the histological findings. After Roux-en-Y reconstruction, she suffered recurrent fever of unknown origin not responsive to several antibiotics (sulbactam/cefoperazone, panipenem/betamipron, piperacillin, amikacin, etc.). When she had a high fever, although C-reactive protein was high $(\sim 10 \mathrm{mg} / \mathrm{dL})$, her aspartate aminotransferase, alanine aminotransferase, lactate dehydrogenase, alkaline phosphatase, gamma-glutamyl transpeptidase, prothrombin time, total bilirubin, direct bilirubin, and immunoglobulin levels were all normal. She had no jaundice, cough, nasal discharge, diarrhea, pyuria, or otitis media. Repeated blood and urine cultures were all negative.
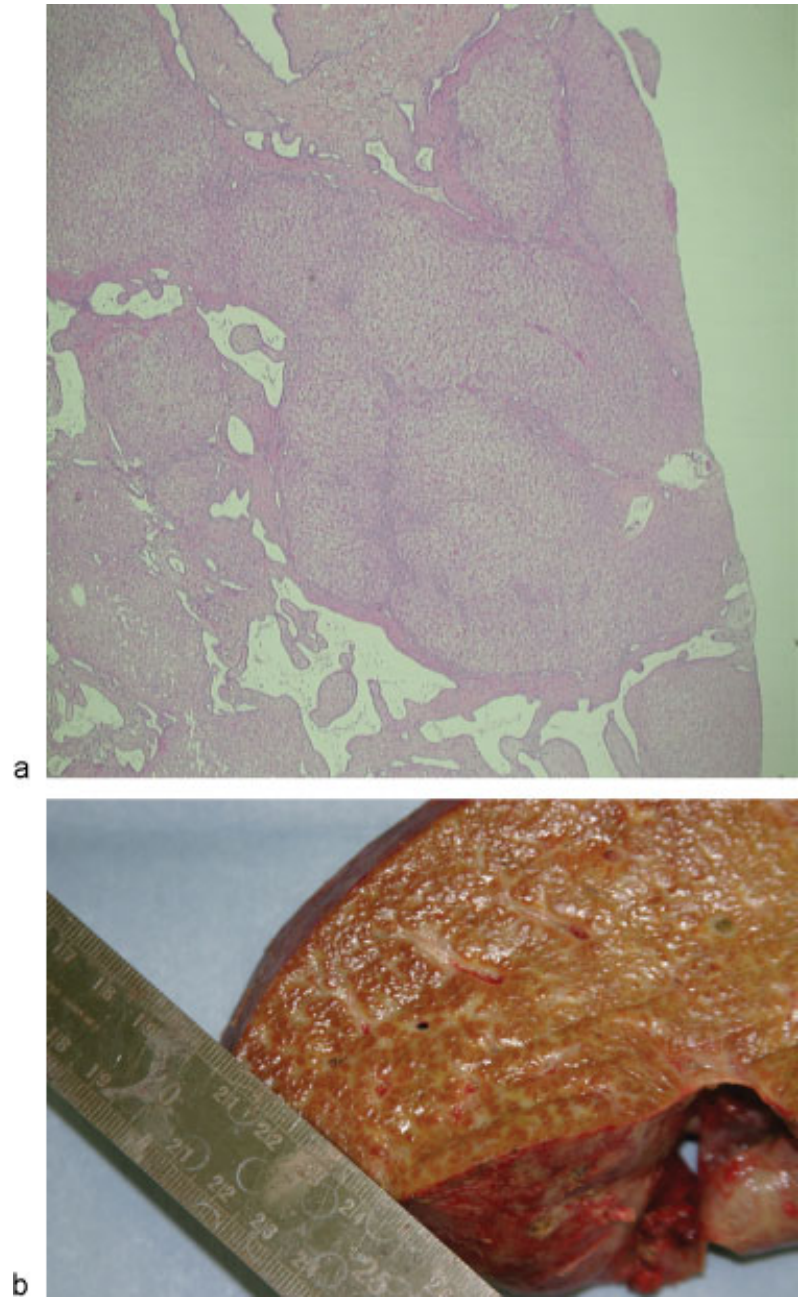

Fig. 2 (a) On histological examination of the liver biopsy specimen, portal areas greatly expanded by fibrous tissue and increased bile ducts are seen. The bands sometimes connect with adjacent portal tracts. Distorted irregular bile ducts are noted in the portal tracts

(hematoxylin and eosin stain). (b) The recipient liver shows dark brown and green.

The symptoms were intractable, and her systemic condition gradually deteriorated and her quality of life was reduced. Therefore, she underwent living donor liver transplantation (LDLT) from her father at the age of 6 years. The recipient liver was dark brown and green ( - Fig. $2 b$ ). A lymph node in the hepatic portal region was enlarged. On histological examination, neutrophilic cholangitis with diffuse infiltration of neutrophils in the bile duct and hepatic vein and reactive lymphadenitis with infiltration of neutrophils and macrophages in sinusoids were seen. Three species of multi-drugresistant Pseudomonas aeruginosa were cultured in the recipient's liver. After LDLT, the patient had no episodes of recurrent fever, and her outcome was good.

\section{Discussion}

In the present case, complete choledochal cyst resection and Roux-en-Y reconstruction were performed in a 4-year-old girl with PBM and a choledochal cyst. At the time of the operation, 
hepatic histological findings were hyperplasia of the interlobular bile duct and diffuse fibril formation around the portal vein, compatible with a diagnosis of CHF. Postoperatively, the patient repeatedly suffered from fever of unknown origin. Although an investigation was performed from various perspectives to identify the cause of the fever, the focus could not be determined. While blood test findings did not include elevated transaminase levels or elevated bile duct-related enzymes such as gamma-glutamyl transpeptidase, it was considered that the cause of the fever was ascending cholangitis with foci at the liver and bile ducts. Therefore, LDLT from the patient's father was performed when the patient was 6 years old. In Japan, the number of deceased donors is still extremely low, especially for pediatric recipients. LDLT was efficacious for a patient with intractable cholangitis following Roux-en-Y reconstruction for CHF and PBM.

Since there are risks of biliary tract carcinoma in patients with PBM and a choledochal cyst, surgery at an early stage is recommended for such cases. ${ }^{5}$ Choledochal cyst resection in Japan is usually performed when the patient is between 1 and 4 years of age. ${ }^{6}$ Therefore, choledochal cyst resection and Roux-en-Y reconstruction were performed when the patient was 4 years old, because PBM was observed in the present case.

CHF can be associated with polycystic kidney, which is a rare disease. It is considered that $\mathrm{CHF}$ and Caroli disease are ductal plate malformations from an embryological point of view. ${ }^{1} \mathrm{CHF}$ is often accompanied by portal hypertension. Even if portal hypertension is clearly present, hepatic function usually remains normal, and the patient's prognosis is relatively good. ${ }^{3}$ Liver transplantation is indicated in those children who develop liver failure in association with portal hypertension. ${ }^{7}$ Also, recurrent cholangitis in CHF is an indication for liver transplantation. ${ }^{8}$ In the present case, the patient suffered repeatedly from intractable cholangitis after RouX-en-Y reconstruction; therefore, this may have caused the liver transplantation to be performed earlier than otherwise. In Alagille syndrome accompanying hypoplasia of the intrahepatic bile duct, performing the Kasai procedure may result in a poor outcome. ${ }^{9}$ One possible mechanism could be that exposure to intestinal contents and/or ascending cholangitis via the portoenterostomy could result in a second hit on already abnormal bile ducts. Therefore, in such cases, the Kasai procedure is not recommended. In ductal plate malformation, as for CHF, the intrahepatic bile duct has a complicated form, and, therefore, once an infection is established, it becomes intractable. Similar to Alagille syndrome, Roux-en-Y reconstruction should be avoided for ductal plate malformations such as CHF. If polycystic kidneys are identified, liver biopsy should be performed before any operative intervention to rule out/diagnose CHF.

\section{Conflict of Interest \\ None}

\section{References}

1 Desmet VJ. Congenital diseases of intrahepatic bile ducts: variations on the theme "ductal plate malformation". Hepatology 1992;16(4):1069-1083

2 Alvarez F, Bernard O, Brunelle F, et al. Congenital hepatic fibrosis in children. J Pediatr 1981;99(3):370-375

3 Kerr DNS, Okonkwo S, Choa RG. Congenital hepatic fibrosis: the long-term prognosis. Gut 1978;19(6):514-520

4 Todani T, Watanabe Y, Urushihara N, et al Choledochal cyst, pancreatobiliary malunion, and cancer. J Hep Bil Pancr Surg 1994; 1(3):247-251

5 Todani T. Congenital choledochal dilatation: classification, clinical features, and long-term results. J Hepatobiliary Pancreat Surg 1997;4:276-282

6 Kobayashi S, Ohnuma N, Yoshida H, et al. Preferable operative age of choledochal dilation types to prevent patients with pancreaticobiliary maljunction from developing biliary tract carcinogenesis. Surgery 2006;139(1):33-38

7 Perisic VN. Long-term studies on congenital hepatic fibrosis in children. Acta Paediatr 1995;84(6):695-696

8 Ko JS, Yi NJ, Suh KS, Seo JK. Pediatric liver transplantation for fibropolycystic liver disease. Pediatr Transplant 2012;16(2): 195-200

9 Kaye AJ, Rand EB, Munoz PS, Spinner NB, Flake AW, Kamath BM. Effect of Kasai procedure on hepatic outcome in Alagille syndrome. J Pediatr Gastroenterol Nutr 2010;51(3):319-321 